\title{
Relevância da roda de conversa no Programa HIPERDIA: foco na alimentação saudável e atividade física
}

\author{
Relevance of the talk wheel in the HIPERDIA Program: focus on healthy diet and physical \\ activity \\ Relevancia de la rueda de conversación en el programa HIPERDIA: foco en la \\ alimentación sana y actividad física
}

Francisco Lucas de Lima Fontes ${ }^{1 *}$, Jadson Farias de Sousa ${ }^{1}$, Karla Raynara Oliveira da Silva ${ }^{1}$, Rayssa Sayuri Rocha Baba², Yvana Marília Sales Medino ${ }^{3}$, Samuel Lopes dos Santos ${ }^{4}$, Yasmin Nunes de Alencar ${ }^{1}$, Onédia Naís de Carvalho ${ }^{2}$, Camila de Araújo Batista ${ }^{1}$, Alayse Gabryelli Vaz de Barros $^{1}$, Samara Sales Gomes de Sousa ${ }^{5}$, Julia Maria dos Santos ${ }^{1}$, Naasson Damasceno Silva ${ }^{1}$, Jéssica Mayra do Nascimento Cabral ${ }^{1}$, Brenda Lícia Martins da Silva1.

\section{RESUMO}

Objetivo: Descrever a experiência acadêmica em uma roda de conversa sobre alimentação saudável e atividade física no Programa HIPERDIA. Relato de experiência: Procurou-se discutir a temática com os participantes da roda de conversa de maneira holística, partindo do pressuposto da realidade financeira de cada um, a importância de garantia de alimentos a todos os sujeitos, as dietas (adesão e fuga), a prática de atividade física atrelada à boa alimentação e exposição da pirâmide alimentar adaptada à população brasileira. Conclusão: Percebeu-se que a roda de conversa possibilitou aos participantes a ampliação do autoconhecimento. A relação acadêmicos-usuários foi bastante positiva. Procurou-se promover uma comunicação dialógica, moldando os conhecimentos expostos pelos participantes ao melhor contexto social e de saúde ao qual cada um estava inserido.

Palavras-chave: Atividade física, Alimentação saudável, Hipertensão arterial sistêmica, Diabetes mellitus.

\begin{abstract}
Objective: To describe the academic experience in a conversation about healthy diet and physical activity in the HIPERDIA Program. Experience report: We tried to discuss the topic with the participants in the conversation in a holistic way, based on the assumption of the financial reality of each one, the importance of guaranteeing food to all subjects, the diets (adherence and flight), practice of physical activity linked to good nutrition and exposure of the food pyramid adapted to the Brazilian population. Conclusion: It was noticed that the conversation wheel enabled the participants to expand self-knowledge. The academic-user relationship was very positive. We sought to promote a dialogical communication, shaping the knowledge exposed by the participants to the best social and health context to which each one was inserted.
\end{abstract}

Keywords: Exercise, Healthy diet, Hypertension, Diabetes mellitus.

\footnotetext{
${ }^{1}$ Faculdade UNINASSAU - Campus Redenção. Teresina, Piauí, Brasil.

*E-mail: lucasfontesenf@hotmail.com

${ }^{2}$ Centro Universitário Uninovafapi. Teresina, Piauí, Brasil.

${ }^{3}$ Centro Universitário Santo Agostinho. Teresina, Piauí, Brasil.

${ }^{4}$ Faculdade FACID - Wyden. Teresina, Piauí, Brasil.

${ }^{5}$ Universidade Federal do Piauí. Teresina, Piauí, Brasil.
} 


\section{RESUMEN}

Objetivo: Describir la experiencia académica en una rueda de conversación sobre alimentación sana y actividad física en el programa HIPERDIA. Relato de experiencia: Se intentó discutir la temática con los participantes de la rueda de conversación de manera holística, partiendo del supuesto de la realidad financiera de cada uno, la importancia de la garantía de alimentos a todos los sujetos, las dietas (adhesión y fuga), la práctica de actividad física vinculada a la buena alimentación y exposición de la pirámide alimentaria adaptada a la población brasileña. Conclusión: Se percibió que la rueda de conversación posibilitó a los participantes la ampliación del autoconocimiento. La relación académicos-usuarios fue bastante positiva. Se buscó promover una comunicación dialógica, moldeando los conocimientos expuestos por los participantes al mejor contexto social y de salud al que cada uno estaba inserto.

Palabras-clave: Ejercicio, Dieta Saludable, Hipertensión, Diabetes mellitus.

\section{INTRODUÇÃO}

Evidências científicas demonstram o crescimento das Doenças Crônicas Não Transmissíveis (DCNTs), associado a diversos fatores de risco, como a alimentação inadequada e desregrada, inatividade física, dislipidemia, tabagismo e uso irracional de álcool (LINDEMANN IL et al., 2016). Na lista das principais DCNTs encontram-se as neoplasias, a diabetes e as doenças cardiovasculares, respiratórias e osteomusculares. Dentre as patologias cardiovasculares, a Hipertensão Arterial Sistêmica (HAS) merece destaque junto com a disfunção metabólica Diabetes Mellitus (DM), doenças que necessitam de um tratamento eficaz e, acima de tudo, de controle rigoroso com relação ao estilo de vida do indivíduo, focando-se, a princípio, nas mudanças de hábitos antes de adentrar com a terapia farmacológica (MELO PKM et al., 2015).

As DCNTs são as principais causas de morte mundialmente, tanto nos países desenvolvidos quanto nos emergentes (BORTOLUZ S, et al., 2016). Em 2011, das mortes registradas no Brasil, $72 \%$ foram por complicações dessas patologias. Estima-se que elas acarretam perda da produtividade laboral e diminuição da renda familiar, com custos de US\$ 4,18 bilhões aos cofres públicos, consequência de complicações de três principais DCNTs: DM, HAS e neoplasias (MALTA et al., 2014).

Calcula-se que, no mundo, a HAS atinja de 20 a 25\% da população (MOURA AA e NOGUEIRA MS, 2013). No Brasil, a doença atinge mais de 30 milhões de pessoas, manifestando-se em $35,8 \%$ dos homens adultos e 30\% das mulheres. Está associada a altos custos socioeconômicos, e compromete a população, a família e o próprio indivíduo portador, ocasionando $40 \%$ de aposentadorias precoces (LIMA DBS et al., 2016).

Mundialmente a DM é responsável por mais de 14\% das mortes por todas as causas. No Brasil, continua sendo um dos principais problemas de saúde pública. Em 2015, segundo dados da International Diabetes Federation, o Brasil ocupava a quarta posição mundial dos países com mais pessoas diabéticas, com 14,3 milhões de pessoas vivendo com a doença. Projeções para 2040 mostram que este número aumentará para 23,3 milhões (SOCIEDADE BRASILEIRA DE DIABETES, 2017).

Aspectos como alimentação e a prática de atividade física influenciam diretamente na prevenção e controle da HAS e da DM. Observa-se atualmente mudanças no padrão nutricional da população mundo afora, não sendo diferente no Brasil, onde destaca-se o consumo imoderado de açúcares, gorduras, alimentos industrializados e produtos ricos em sódio, além da redução na ingesta de feijão e arroz, frutas e vegetais (BRASIL, 2012).

A prática de atividade física regularmente é orientada ao tratamento da HAS e da DM, pois trata-se de uma conduta não farmacológica e de promoção da qualidade de vida, ao passo em que trabalha na diminuição dos níveis pressóricos e garante melhor controle glicêmico dos sujeitos (REIS HHT e MARTINS JCB, 2017). Pessoas sedentárias e menos condicionadas possuem o dobro de chances de desenvolverem complicações cardiovasculares, se comparados aos indivíduos ativos e condicionados (VIEIRA CPB et al., 2016). 
Em nosso país, a implantação do Sistema Único de Saúde (SUS) no ano de 1990 trouxe significativas mudanças no modelo de organização do serviço público de saúde, fortalecendo, a princípio, a atenção básica e anos mais tarde solidificando o Programa de Saúde da Família. A Estratégia de Saúde da Família (ESF), como é conhecida atualmente o antigo programa, é considerada porta de entrada do sistema de saúde no Brasil (SZWARCWALD CL et al., 2013).

Dentre as principais responsabilidades da ESF estão o controle da HAS e da DM. Visando uma assistência integral à população atingida por estas duas patologias, foi criado em 2001 o Plano de Reorganização da Atenção à Hipertensão Arterial e Diabetes Mellitus (PRAHADM), que daria origem ao Programa HIPERDIA. Tal programa permite o cadastro e acompanhamento de pacientes com HAS/DM, captados e vinculados às ESF ou equipes de atenção básica do SUS. O objetivo deste programa é possibilitar o aumento da longevidade na população brasileira por meio de ações capazes de diminuir a morbimortalidade por HAS/DM e garantir melhoria da qualidade de vida (BEZERRA GC et al., 2015; RAMOS VKS et al., 2014).

Diante disso, o objetivo do presente estudo foi descrever a experiência acadêmica em uma roda de conversa sobre alimentação saudável e atividade física no Programa HIPERDIA.

\section{RELATO DE EXPERIÊNCIA}

O relato foi vivenciado ainda durante a formação acadêmica, no nono período do curso bacharelado em Enfermagem de uma instituição de ensino superior privada de Teresina, capital do Piauí. O plano de ação foi desenvolvido por meio das atividades práticas da disciplina de "Estágio Supervisionado I" no decurso do segundo semestre de 2017.

O "Estágio Supervisionado I" tem suas atividades desenvolvidas em unidade básica de saúde. Na prática, os alunos têm a oportunidade de integração maior com a ESF e com programas da rede básica dos serviços de saúde, de prestar assistência à mulher, criança, adolescente, adultos e idoso no âmbito da atenção primária à saúde por meio da consulta de Enfermagem, de executar ações de educação em saúde, de exercer as atividades gerenciais e assistenciais do enfermeiro na unidade, de participar das atividades promovidas pela unidade de saúde, de realização de visitas domiciliares e acompanhamento da imunização durante as consultas.

A roda de conversa foi ambientada no local de espera para atendimento, ao qual podemos chamar de "sala de espera", de uma ESF, localizada na Zona Sul da capital piauiense.

Para a coleta dos dados deste estudo foi planejada e executada uma roda de conversa sobre alimentação saudável com pacientes cadastrados no Programa HIPERDIA. Participaram da atividade 17 usuários, de ambos os sexos, sendo 14 mulheres e 3 homens, com idades variadas. $O$ ambiente foi organizado de modo que todos os participantes se sentissem à vontade.

A atividade foi realizada no turno da manhã. Foram expostos cartazes confeccionados pelos próprios acadêmicos aos participantes abordando temas, como: "Comida de verdade", "Alimentação saudável com o que se tem em casa", "Pirâmide alimentar", "Atividade física".

Iniciou-se a roda de conversa com a exposição de cartazes elaborados pelos próprios acadêmicos. Os cartazes continham gravuras e frases que referiam à busca por uma alimentação saudável. Procurou-se discutir a temática partindo do pressuposto da realidade financeira de cada um dos participantes, enfatizando a importância da ingesta de alimentos mais acessíveis economicamente, como o bom e velho feijão com arroz, determinados tipos de frutas e acompanhamentos aos grãos citados anteriormente.

Parte dos participantes evidenciou certa relutância na adesão às dietas orientadas pelos profissionais de saúde. Para eles, a alimentação por si só é cara e "comer bem nem sempre é possível". Muitos referiram fuga às dietas de maneira recorrente, apesar de afirmarem a tentativa de aderência a uma alimentação saudável.

Foi questionada durante a roda de conversa a prática de atividade física. Muitos admitiram não praticar atividade física pela falta de tempo. Como grande parte dos participantes do relato era do sexo feminino, as

REAS/EJCH | Vol. Sup. 23 | e394 | DOI: https://doi.org/10.25248/reas.e394.2019 Página 3 de 6 
mulheres presentes atribuíram atividades como cuidar da casa, dos filhos/netos e outras responsabilidades como sendo os motivos pelos quais não praticavam atividade física de maneira regular.

Dos 17 participantes apenas dois referiram a prática regular de atividade física, chamando atenção para o discurso de um dos presentes, de que passou a se cuidar mais depois que foi internado com fortes dores no peito. Outros narraram ainda que não realizam atividade física devido a limitação da sua própria patologia e/ou alguma outra afecção.

\section{DISCUSSÃO}

O recurso da roda de conversa coletiva baseia-se na formação de espaços de diálogo, na qual pessoas se expressam, escutam os outros e a si mesmas. Esta metodologia instiga a criação de autonomia por parte dos participantes por meio do mecanismo da problematização, da troca de experiências e da reflexão para a ação (CAMPOS GWS, 2000). O profissional de saúde, durante a roda de conversa, deve atuar como um facilitador, preocupando-se com a sua postura, sua linguagem, com o material de apoio utilizado e com o local da ação, pois essas particularidades devem ser adaptadas ao padrão teórico, ao assunto da roda e ao grupo ao qual se destina (MANDRÁ PP e SILVEIRA FDF, 2013).

Grande parte das DCNTs pode ser evitável por meio da adoção de um estilo de vida saudável. Práticas nocivas incluem alimentação inadequada, ligeiramente ligada a doenças como diabetes e patologias cardiovasculares (FITZGERALD N et al., 2013). O Brasil ainda vivencia o período de transição nutricional, descrita por obesidade, desnutrição e carências de determinados nutrientes que favorecem o surgimento de outras doenças (SOUSA EB, 2010).

Gomes HMS e Teixeira EMB (2016) pautam a importância de garantia de alimentos a todos os sujeitos, com qualidade, quantidades aceitáveis às necessidades humanas básicas, de maneira constante e sem prejudicar outras necessidades fundamentais à manutenção de uma vida digna e saudável.

Da perspectiva econômica, percebe-se que a renda colabora significativamente para complicações nutricionais, levando em conta que a limitação financeira impede e atrapalha a prática de uma alimentação saudável que englobe regularmente a ingesta de frutas, vegetais, cereais integrais, bem como a utilização de carne, leite e derivados, que configuram em consideráveis gastos, frente a outros custos e necessidades familiares (FRAZZIO DMG, 2012).

Dessa forma, alimentos com alta densidade energética e elevadas taxas de gordura e açúcares, mais acessíveis economicamente, prevalecem às condições financeiras em detrimento de alimentos saudáveis e de maior teor nutritivo (VASCONCELOS SML, 2015).

Outros fatores que dificultam na aderência às orientações dietoterápicas repassadas pelo profissional de saúde estão relacionados a aspectos como mudança de hábitos inadequados, cumprimento de horários, valor cultural dos alimentos, limitações socioeconômicas e fatores psicológicos (PINHO L et al., 2012).

Uma ação realizada em outro estudo expôs as dificuldades de autocuidado em pacientes hipertensos. Nela, percebeu-se que os participantes buscavam aderir às boas práticas de saúde, mas enfrentavam desafios em alterar o estilo de vida, especialmente em relação à execução de atividade física (FONTES FLL e SANTANA RS, 2018).

Um dos fatores que dificultam a prática de atividade física é a "dor" que esta produz (BRITO DMS, et al., 2008), demonstrado pelos presentes por desestímulo e procrastinação. O estudo de Silva FO et al. (2015) mostrou que a prática de atividade física entre sujeitos cadastrados no Programa HIPERDIA foi maior em mulheres $(47,5 \%)$ ante apenas $8,3 \%$ de homens ativos. Esses dados ajudam a imprimir a imagem de que as mulheres se preocupam mais com a saúde, quando comparadas aos homens.

Azevedo SS e Cruz RS (2017) explanam que o tempo em que o sujeito se desprende da vida ativa de atividades físicas e passa a ser sedentário propicia o desenvolvimento de doenças, diminui a expectativa e qualidade de vida e aumenta o risco de mortalidade. O estilo de vida contemporâneo torna as pessoas

REAS/EJCH | Vol. Sup. 23 | e394 | DOI: https://doi.org/10.25248/reas.e394.2019 Página 4 de 6 
sedentárias por conta do trabalho, da renda, da classe social e da percepção de mundo sobre o processo saúde-doença.

A prática de atividade física associada com a alimentação saudável são ações essenciais no controle de doenças, como a HAS e a DM, além de garantir um bem-estar físico e mental. Para se obter efetivo controle de tais patologias são necessárias ações de Educação em Saúde com a população, com incentivo dos indivíduos a práticas de autocuidado, tornando-os coparticipantes no processo saúde-doença (FERREIRA N, et al., 2009).

A alimentação saudável deve ser idealizada com alimentos dos mais diversos grupos alimentares, de procedência segura e conhecida, com ingestão em horários definidos, considerando-se as singularidades físicas, sociais e emocionais, de forma a executar as orientações nutricionais. No Brasil, os recursos mais usados para norteio alimentar são o Guia Alimentar Para População Brasileira, do Ministério da Saúde, e a Pirâmide Alimentar adaptada, da investigadora Sônia Tuncunduva Philippi. Essa pirâmide resguarda condutas essenciais a uma alimentação saudável: variedade, equilíbrio e moderação (GOMES HMS e TEIXEIRA EMB, 2016).

\section{CONSIDERAÇÕES FINAIS}

Durante a roda de conversa, os sujeitos cadastrados no Programa HIPERDIA tiveram a oportunidade de compartilhar com os outros a experiência com suas patologias e a busca por uma alimentação saudável na medida do possível. Percebeu-se que a roda de conversa possibilitou aos participantes a ampliação do autoconhecimento. A relação acadêmicos-usuários mostrou-se positiva. Como detentores de um saber científico, não houve em nenhum momento, por parte dos acadêmicos, inferiorização do conhecimento empírico/vulgar dos usuários, procurou-se pois promover uma comunicação dialógica, moldando tais conhecimentos ao melhor contexto social e de saúde ao qual cada participante do Programa HIPERDIA estava inserido, com vistas a garantir uma melhor qualidade de vida individual e coletiva. Ações como roda de conversa, palestras, dinâmicas e oficinas no âmbito da Estratégia de Saúde da Família são de suma importância para que a equipe multiprofissional conheça a realidade de sua população adscrita, identificando problemas e buscando soluções, tendo assim a oportunidade de planejar estratégias e em seguida implementá-las.

\section{REFERÊNCIAS}

1. AZEVEDO SS, CRUZ RS. Fatores socio antropológicos que influenciam no comportamento sedentário. Revista Ciências e Saberes, 2017; 3(4): 671-675.

2. BEZERRA GC et al. Implementação do grupo HIPERDIA em uma unidade de saúde da família: um relato de experiência. Revista Enfermagem Digital Cuidado e Promoção da Saúde, 2015; 1(1): 19-22.

3. BORTOLUZ S et al. Condições de saúde e utilização de um serviço de atenção primária em pacientes hipertensos e/ou diabéticos. Ciências \& Saúde, 2016; 9(3): 156-166.

4. BRASIL. Ministério da Saúde. Secretaria de Atenção à Saúde. Política Nacional de Alimentação e Nutrição. Brasília: Ministério da Saúde, 2012.

5. BRITO DMS et al. Qualidade de vida e percepção da doença entre portadores de hipertensão arterial. Caderno de Saúde Pública, 2008; 24(4): 933-940.

6. CAMPOS GWS. Um método para análise e cogestão de coletivos. 1ª ed. São Paulo: Hucitec, 2000.

7. FERREIRA N et al. Promoção da saúde com ênfase na atividade física e alimentação saudável. Cadernos UniFOA, 2009; 4(1): 91-96.

8. FITZGERALD N et al. Practice Paper of the Academy of Nutrition and Dietetics: The role of nutrition in health promotion and disease prevention. Journal of the Academy of Nutrition and Dietetics, 2013; 113(7): 972-979.

9. FONTES FLL, SANTANA RS. Dificuldades de autocuidado em pacientes hipertensos de uma Estratégia de Saúde da Família. Revista de Enfermagem da UFPI, 2018; 7(2): 90-94.

10. FRAZZIO DMG. Envelhecimento e qualidade de vida - uma abordagem nutricional e alimentar. Revisa, 2012; 1(1): 76-88.

REAS/EJCH | Vol. Sup. 23 | e394 | DOI: https://doi.org/10.25248/reas.e394.2019 Página $\mathbf{5}$ de $\mathbf{6}$ 
11. GOMES HMS, TEIXEIRA EMB. Pirâmide alimentar: guia para alimentação saudável. Boletim Técnico IFTM, 2016; 2(3): 10-15.

12. LINDEMANN IL et al. Dificuldades para alimentação saudável entre usuários da atenção básica em saúde e fatores associados. Ciência \& Saúde Coletiva, 2016; 21(2): 599-610.

13. LIMA DBS et al. Associação entre adesão ao tratamento e tipos de complicações cardiovasculares em pessoas com hipertensão arterial. Texto \& Contexto Enfermagem 2016; 25(3): 1-9.

14. MANDRÁ PP, SILVEIRA FDF. Satisfação de usuários com um programa de roda de conversa em sala de espera. Audiology - Communication Research, 2013; 18(3): 186-193.

15. MELO PKM et al. Orientação sobre o cuidado com alimentação dos idosos que pertencem ao grupo de HIPERDIA: experiência em serviço. Anais do Congresso Internacional de Envelhecimento Humano, 2015; 2(1): 1-6.

16. MOURA AA, NOGUEIRA MS. Enfermagem e educação em saúde de hipertensos: revisão da literatura. Journal of Management \& Primary Health Care, 2013; 4(1): 36-41.

17. PINHO L et al. Percepções de hipertensos sobre o acompanhamento nutricional recebido em um Núcleo de Apoio à Saúde da Família (NASF). Motricidade, 2012; 8(s2): 58-66.

18. RAMOS VKS et al. Caracterização dos usuários do HIPERDIA em uma unidade básica de saúde em um município do Estado do Maranhão. Revista de Investigação Biomédica, 2014; 6(1): 86-95.

19. REIS HHT, MARTINS JCB. Nível de atividade física de diabéticos e hipertensos atendidos em um centro hiperdia. Arquivos de Ciências da Saúde, 2017; 24(3): 25-30.

20. SILVA FO et al. Perfil de pacientes cadastrados no HIPERDIA: conhecendo o estilo de vida. Revista de Saúde Coletiva da UEFS, 2015; 5(1): 33-39.

21. SOCIEDADE BRASILEIRA DE DIABETES. Diretrizes da Sociedade Brasileira de Diabetes 2017-2018. São Paulo: Editora Clannad, 2017.

22. SOUZA EB. Transição nutricional no Brasil: análise dos principais fatores. Cadernos UniFOA, 2010; 5(13): 49-53.

23. SZWARCWALD CL et al. Recommendations and practice of healthy behaviors among patients with diagnosis and diabetes in Brazil: National Health Survey (PNS), 2013. Revista Brasileira de Epidemiologia, 2015; 18(2): $132-145$.

24. VASCONCELOS SML et al. Insegurança Alimentar em Domicílios de Indivíduos Portadores de Hipertensão e/ou Diabetes. Internacional Journal of Cardiovascular Sciences, 2015; 28(2):114-121.

25. VIEIRA CPB et al. Prevalência referida, fatores de risco e controle da hipertensão arterial em idosos. Ciência, Cuidado e Saúde, 2016; 15(3): 413-420. 